Udo Böhm

\section{ÜBERSICHTEN/ REVIEWS}

\section{Mechanismen für die Ent- stehung der essenziellen Hypertonie}

Beginnen wir mit den von der Hochdruckliga aufgeführten „klassischen“ Faktoren, die als Auslöser für die primäre Hypertonie fungieren. Hierzu zählen

- Genetik und Alter,

- Nikotinabusus,

- Alkoholabusus,

n Übergewicht,

- Bewegungsmangel,

- Medikamente (z.B. nicht steroidale Antirheumatika, Nasentropfen, Antibabypille, Appetitzügler),

n ungesunde Ernährungsweise (mit hohem Anteil an Fleisch und Kochsalz sowie mit wenig Obst und Gemüse).

Einen sehr aktuellen Risikofaktor stellen chronische psychosoziale Stressbelastungen dar, bei denen über eine anhaltende Aktivierung des Sympathikus und Störungen der sog. neuroendokrinen Achse eine Erhöhung des Blutdrucks erfolgen kann.

Die Auflistung zeigt, dass der primäre Hochdruck wohl v.a. eine Folge eines ungünstigen persönlichen Lebensstils ist.

Die Pathomechanismen bei der Entwicklung des Hypertonus umfassen zunächst eine chronische Überaktivität des Sympathikus gepaart mit einer erhöhten Ausschüttung von Renin und anderen Neurohormonen (z.B. antidiuretisches Hormon, Endothelin, Zytokine, Thromboxan $\left.A_{2} / B_{2}\right)$ sowie einer Inhibierung von vasoak-

\title{
Hypertonie und Orthomolekularmedizin
}

\section{Zusammenfassung}

Die Hypertonie ist eine komplexe und langfristig nur schwer zu behandelnde Erkrankung mit einem hohen Risiko für Folgeschäden. Man unterscheidet zwischen der primären oder essenziellen Hypertonie und dem sekundären Hypertonus, der z.B. durch Arteriosklerose oder Erkrankungen der Niere ausgelöst werden kann. Der Artikel zeigt neben der Pathogenese der primären Hypertonie, den Risikofaktoren und der pharmakologischen Therapie die präventiven und therapeutischen Möglichkeiten der Orthomolekularmedizin auf.

\section{Schlüsselwörter}

Hypertonie, Orthomolekularmedizin, Risikofaktoren, Lebensstil, Ernährung, Mikronährstoffe.

\section{Abstract}

Hypertension is a complex disease with a high risk of secondary damages, which is difficult to treat in the long term. It has to be distinguished between primary or essential hypertension and the secondary hypertonus, which can be caused for example by arteriosclerosis or diseases of the kidneys. The article shows the preventive and therapeutic options of orthomolecular medicine in addition to the pathogenesis of primary hypertension, the risk factors, and the pharmacological therapy.

\section{Keywords}

Hypertension, orthomolecular medicine, risk factors, life style, nutrition, micronutrients.

tiven Substanzen (z.B. Stickstoffmonoxid, Bradykinin, Substanz P, natriuretische Peptide).

Vereinfacht dargestellt führen diese Abläufe zu einem Anstieg der Spiegel von Angiotensin-Converting-Enzym (ACE), Angiotensin I, Angiotensin II und Aldosteron mit erhöhter Natriumeinlagerung und erhöhter Kaliumausscheidung, zu einer Zunahme an Blutvolumen, Herzfrequenz und Herzkontraktiliät sowie zu Stickstoffmonoxidmangel (NO-Mangel), Vasokonstriktion, endothelialer Dysfunktion, Herzhypertrophie und Anstieg des Herzzeitvolumens. Neue
Untersuchungen bestätigen zudem den hohen Stellenwert von freien Radikalen [711] und chronischen Entzündungsprozessen bei diesen Vorgängen und insbesondere bei der Entstehung von endothelialer Dysfunktion und relativem Stickstoffmonoxidmangel mit verminderter Vasodilatation (Abb.1). Oxidativer Stress kann nach dem heutigen Wissensstand sowohl eine Hypertonie auslösen als auch als Folge einer Hypertonie auftreten. 


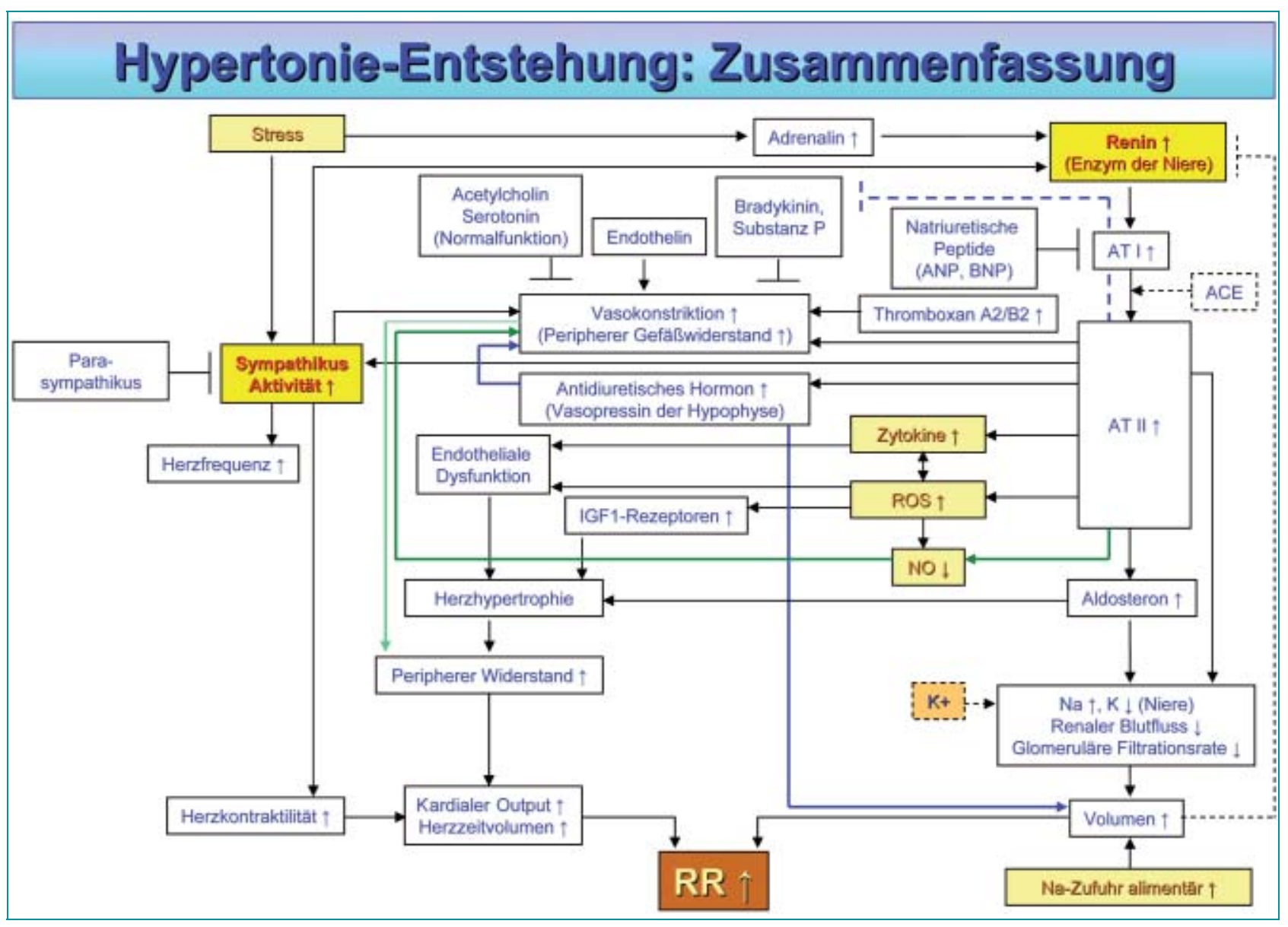

Abb. 1: Grafische Darstellung der Entstehung einer Hypertonie. (c) Udo Böhm

\section{Nicht medikamentöse Prävention und Therapie}

Eine effektive Prävention der essenziellen Hypertonie, aber auch eine signifikante Reduzierung des Risikos für sekundäre Hypertonieformen ist v.a. durch die sich aus den o.g. Risikofaktoren ergebende $\mathbf{0 p}$ timierung des persönlichen Lebensstils zu erwarten. Es ist nicht sonderlich erstaunlich, dass eine Kombination der Einzelmaßnahmen auch therapeutisch Ergebnisse bringt, die einer „klassischen“ optimierten medikamentösen Hochdrucktherapie mindestens gleichwertig sind.

Einen gewichtigen Anteil am Erfolg der Lebensstilmedizin hat eine frische, regionale, vollwertige und kalorienangepasste Ernährung nach dem Vorbild der sog. traditionellen mediterranen Kost [1-5]. Sie könnte aus Sicht der Orthomolekularmedizin zunächst eine bei Hypertonikern häufig auffindbare Mikronährstoffunterversorgung verhindern [6] und die Basis für eine auf Dauer ausreichende Zufuhr aller essenziellen Mikronährstoffe liefern, die sowohl für die allgemeine Gesundheit als auch für die Prävention und Therapie von Hypertonie unverzichtbar sind (Abb. 2). Außerdem

- hilft sie bei der Vermeidung oder der Behebung von Übergewicht,

- verringert Entzündungen und oxidative Belastungen,

- verbessert die allgemeine Stoffwechselsituation und

- reduziert eine Volumenzunahme durch Einschränkung der Kochsalzzufuhr bzw. eine Erhöhung des Verhältnisses zwischen Kalium und Natrium.

\section{Klassische medikamentöse} Therapie

Die klassische universitäre Therapie der essenziellen Hypertonie nutzt derzeit v.a. Thiaziddiuretika, Beta-Blocker, KalziumAntagonisten, ACE-Hemmer und AT2-Ant- agonisten, Alpha-1-Blocker, zentral wirkende Antisympathikotonika und Aldosteronantagonisten.

Die Wirksamkeit der einzelnen Stoffgruppen wird neben vielen Studien mit einer unterschiedlichen Beeinflussung der o.g. Pathomechanismen begründet. Alle Substanzen haben individuell unterschiedliche Responderraten, kommen - auch abhängig von den Vorlieben des einzelnen Arztes - entweder als Monotherapie oder als Kombination aus mehreren Gruppen zum Einsatz, sind auf lange Sicht häufig nicht erfolgreich und können zudem schwerwiegende Nebenwirkungen produzieren.

\section{Prävention und Therapie mit Mikronährstoffen}

Lassen Sie mich deshalb auf die Mikronährstoffmedizin eingehen und die Frage beantworten, ob sie bei Hypertonie als Ergänzung oder Alternative zur „klassischen 


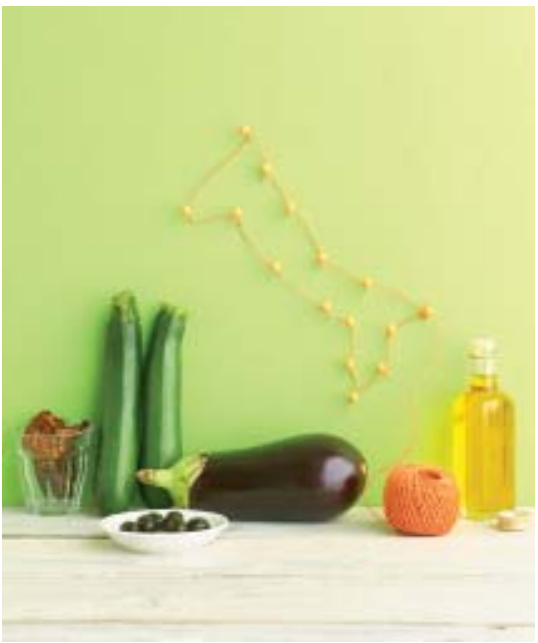

Abb. 2: Großen Anteil am Erfolg der Lebensstilmedizin hat die richtige Ernährung. Sie sollte sich am Vorbild der traditionellen mediterranen Kost orientieren. @ Meike Bergmann/TVG

medikamentösen Therapie“ nützlich sein kann: Als Begründung für ihren Einsatz wäre es natürlich wünschenswert, wenn die für die klassischen Medikamente angedachten Wirkmechanismen auch beim Einsatz von Mikronährstoffen vorgefunden würden, wie

- Verringerung der Sympathikusaktivität bzw. Einstellung eines Gleichgewichts zwischen Sympathikus und Parasympathikus,

- Reduzierung der Ausschüttung von Renin sowie ACE, Angiotensin I, Angiotensin II und Aldosteron,

- Verringerung der Vasokonstriktion und Verbesserung der NO-Verfügbarkeit,

- Erhöhung der Kaliumverfügbarkeit.

Diese Mechanismen sollten soweit möglich durch weitere positive Effekte unterstützt werden wie:

- Verbesserung der Endothelfunktion,

- Reduzierung von Radikalbelastung und Inflammation,

- Reduzierung des Arterioskleroserisikos (z.B. Inhibierung der Lipidperoxidation).

Falls Mikronährstoffe diese Basisbedingungen in der Theorie erfüllen können, sollten sie natürlich durch entsprechende Studien mit Evidenzcharakter unterstützt werden.

Ich bin davon überzeugt, dass beide Voraussetzungen erfüllt werden, und stelle $\mathrm{zu}$ diesem Zweck die bei Hypertonie geeigneten Leitsubstanzen der Mikronährstoffme- dizin, ihre wichtigsten Wirkungsmechanismen und Hinweise auf positive Studien in einer tabellarischen Übersicht dar (Tab.1).

Die den vorgestellten Mikronährstoffen jeweils zur Seite gestellten Studienbeispiele zeigen, dass sich die erwarteten biochemischen Mechanismen auch in der praktischen Umsetzung bewähren und Mikronährstoffe einerseits präventiv die Prävalenz von Hypertonie senken können und andererseits als Erweiterung des bestehenden Therapiespektrums mit einer sehr geringen Nebenwirkungsquote angesehen werden können. Selbst wenn sie allein nicht für eine optimale Blutdruckeinstellung ausreichen sollten, können sie zumindest signifikant dazu beitragen, Dosis und Nebenwirkungsrisiko der klassischen Hypertoniebehandlung zu reduzieren.

\section{Diagnostik}

Ich empfehle zunächst die übliche Standarddiagnostik. Hierzu zählen neben der klinischen Untersuchung mit Messung von Blutdruck und BMI auch EKG, 24-Stunden-Blutdruckmessung, Ergometrie, Sonografie (Herz, Oberbauch) und ein Standardbasislabor (mit BB, Kreatinin, Kalium, Glukose, Natrium, Harnsäure, Lipiden, $\gamma-G T$, Urinstatus, Mikroalbumin im Urin, TSH).

Besonders wichtig erscheint mir aber eine detaillierte Familien-, Lebensstil-, Medikamenten-, Berufs- und Ernährungsanamnese, denn nur mithilfe dieser Daten habe ich später eine echte Chance, die Lebensstilrisiken und die Krankheit Hypertonie auf Dauer effizient zu behandeln.

Vonseiten der Orthomolekularmedizin bedarf es zunächst keiner besonderen $\mathrm{Zu}$ satzuntersuchungen: Wir behandeln (wie es in der Schulmedizin üblich ist) zunächst mit den laut Biochemie und Studien am wahrscheinlichsten wirksamen Substanzen und ergänzen bzw. ändern die Therapie bez. Stoffzusammensetzung und Dosierung nach Bedarf, falls keine ausreichende Wirkung eintreten sollte (Abb. 3). In besonderen Fällen kann man natürlich eine orthomolekularmedizinische Zusatzlabordiagnostik integrieren, mit Spiegelbestimmungen der Leitmikronährstoffe (um z.B. die individuelle Dosierung von Vitamin D festle- gen zu können), mit Untersuchungen bez. oxidativem und nitrosativem Stress, Entzündungen, Homocysteinerhöhung oder Störungen des Säure-Basen-Haushalts sowie Leistung des Immunsystems, der Mitochondrien, der neuroendokrinen Achse usw.

\section{Praxis}

Am Beispiel eines ärztlichen Kollegen möchte ich kurz darstellen, wie man eine orthomolekularmedizinische Behandlung der Hypertonie einleiten kann.

\section{Kollege M.J., 56 Jahre}

Oberarzt in einer internistischen Klinik, hat einen Blutdruck von konstant mind. 155/ $100 \mathrm{mmHg}$, einen BMI von 28, isst viel und unkontrolliert, trinkt zu viel Bier, nimmt sich keine Zeit für Sport, hat ein Kind in der Pubertät und lebt getrennt. Er klagt über chronischen beruflichen und privaten Stress. Er war immer gesund. In der Familie gibt es keine Häufung kardiovaskulärer Erkrankungen.

In der umfangreichen, klassischen Technik- und Labordiagnostik ergeben sich außer einer Erhöhung von LDL und einer Erniedrigung von HDL sowie einem pathologischen Glukosetoleranztest keine wesentlichen Auffälligkeiten. Der Vitamin-D-Spiegel ist niedrig bei $21 \mathrm{ng} / \mathrm{ml}$.

Ich beginne die Therapie leitliniengerecht mit einer Beratung zur konsequenten Lebensstilanpassung, die ich aber an dieser Stelle nicht im Detail vorstellen kann. Zusätzlich rezeptiere ich eine initiale Kombinationstherapie mit Mikronährstoffen, bei der ich mich an der Studienlage orientiere, und mit der ich v.a. die wichtigsten Pathomechanismen bedienen und das Risiko einer Lipidperoxidation reduzieren möchte (Tab.2).

Ich werde nun die Entwicklung der Blutdruckwerte 4 Wochen lang beobachten. Falls sie sich nicht zufriedenstellend verändern sollten, müssen wir unsere Therapie anpassen. Es wäre dann sinnvoll, als nächstes zusätzlich Coenzym Q10 und Kalium (insbesondere bei niedrigen Spiegeln) sowie später auch Vitamin $B_{1}$, Vitamin $B_{3}$ und Folsäure zu testen. 
Tab. 1: Leitsubstanzen der Mikronährstoffmedizin bei Hypertonie und ihre wichtigsten Wirkungsmechanismen.

\begin{tabular}{|c|c|c|}
\hline Substanz & Wirkungsmechanismen & Studien \\
\hline Vitamin C & $\begin{array}{l}\text { Antioxidans; reduziert oxidativen Stress und verbessert die Verfügbarkeit des durch L-Arginin induzierten NO. } \\
\text { Es wirkt auch blutdrucksenkend. }\end{array}$ & [12-14] \\
\hline Vitamin D & $\begin{array}{l}\text { Wirkt antiinflammatorisch und agiert als endokriner Suppressor der Reninbiosynthese; niedrige Vitamin-D- } \\
\text { Spiegel erhöhen bei Hypertonikern das kardiovaskuläre Risiko. }\end{array}$ & {$[15-18]$} \\
\hline B-Vitamine & $\begin{array}{l}\mathrm{B}_{1}, \mathrm{~B}_{3} \text { und Folsäure reduzieren den Blutdruck; } \mathrm{B}_{1} \text { und } \mathrm{B}_{3} \text { wirken dabei v. a. vasodilatierend, Folsäure verbessert } \\
\text { die Barorezeptorsensitivität und die vagale Kontrolle des Blutdrucks. Ein weiterer bekannter Effekt von Folsäure } \\
\text { ist die Senkung von Homocystein (gemeinsam mit Vitamin } B_{6} \text { und } B_{12} \text { ). Verschiedene Studien weisen auf } \\
\text { eine Blutdrucksteigernde Wirkung von Homocystein hin und zeigen eine Reduzierung des Blutdrucks durch } \\
\text { Senkung erhöhter Homocysteinspiegel. Allerdings ist nicht ganz klar, ob für die Blutdrucksenkung eine } \\
\text { Reduzierung der Homocysteinspiegel oder die Wirkung der B-Vitamine alleine verantwortlich sind. }\end{array}$ & {$[19-24]$} \\
\hline Vitamin $\mathrm{E}$ & Antioxidans; reduziert das erhöhte LDL-Oxidations-Risiko bei Hypertonikern. & [25] \\
\hline L-Arginin & $\begin{array}{l}\text { Reduziert den Blutdruck. Muttersubstanz von Stickstoffmonoxid und Substrat der NO-Synthase, deshalb wich- } \\
\text { tig für Funktion des Endothels und für Gefäßweite; inhibiert das Reninsystem, senkt ACE und Angiotensin II. }\end{array}$ & [26-29] \\
\hline Coenzym Q10 & $\begin{array}{l}\text { Senkt den Blutdruck, wichtig für Energiehaushalt und antioxidatives System, trägt zur Verbesserung der } \\
\text { Endothelfunktion bei. }\end{array}$ & [30-33] \\
\hline Kalium & $\begin{array}{l}\text { Reduziert Blutdruck und fördert Parasympathikusaktivität; hohe Kochsalzzufuhr und Thiaziddiuretika } \\
\text { reduzieren Kaliumspiegel. Hohe Kaliumzufuhr reduziert negative Auswirkungen von Natrium, weshalb auf } \\
\text { ein ausgewogenes Verhältnis von Kalium zu Natrium geachtet werden sollte. }\end{array}$ & [34-37] \\
\hline Magnesium & $\begin{array}{l}\text { Senkt Blutdruck und Natrium, reduziert Stressbelastungen und Sympathikusaktivität, fördert Vasodilatation } \\
\text { und Parasympathikus. }\end{array}$ & [38-43] \\
\hline $\begin{array}{l}\text { Omega-3-Fett- } \\
\text { säuren }\end{array}$ & $\begin{array}{l}\text { Reduzieren Blutdruck, regulieren den vasomotorischen Tonus und die renale Salzexkretion, reduzieren } \\
\text { Vasokonstriktorenproduktion, ACE-Aktivität, Bildung von Angiotensin II und TGF- } \beta \text {-Expression sowie das } \\
\text { vasokonstriktive, arrhythmogene, proaggregatorische Thromboxan } \mathrm{A}_{2} / \mathrm{B}_{2} \text { und proinflammatorische } \\
\text { Prostaglandine; sie erhöhen die NO-Bildung, aktivieren den Parasympathikus, verbessern die Vasodilatation } \\
\text { und wirken antientzündlich. }\end{array}$ & [44-46] \\
\hline $\begin{array}{l}\text { Sekundäre } \\
\text { Pflanzenstoffe }\end{array}$ & $\begin{array}{l}\text { Vor allem die Polyphenole (z.B. in Rotwein, Olivenöl, grünem Tee oder Soja) reduzieren den Blutdruck. Sie } \\
\text { wirken antioxidativ und antientzündlich, reduzieren ADMA, CRP und oxidiertes LDL, induzieren die eNOS für } \\
\text { die NO-Bildung und verbessern dadurch Endothelfunktion und Vasodilatation. Besonders gut untersucht ist } \\
\text { das Salvestrol Resveratrol, das sich insbesondere in Rotweinen findet. Es reduziert zusätzlich Angiotensin II } \\
\text { und den Vasokonstriktor Endothelin 1. Der vielfältige Nutzen sekundärer Pflanzenstoffe erklärt auch einen } \\
\text { Teil der Wirkung der traditionellen mediterranen Kost. }\end{array}$ & [47-65] \\
\hline $\begin{array}{l}\text { Phenylalanin, } \\
\text { Tyrosin, } \\
\text { L-Tryptophan }\end{array}$ & $\begin{array}{l}\text { Muttersubstanzen von Dopamin, Adrenalin und Noradrenalin bzw. Serotonin. Es gibt keine aussagekräftigen } \\
\text { Studien für diese Stoffe im direkten Zusammenhang mit der Hypertonie. Bei chronischem Stress aber kann } \\
\text { die Bildung von Stresshormonen, Cortisol und Serotonin eingeschränkt sein. Die Zufuhr dieser Aminosäuren } \\
\text { kann dann die gestörte oder gar inaktive neuroendokrine Stressachse wieder aktivieren. }\end{array}$ & \\
\hline Sonstiges & $\begin{array}{l}\text { Nicht vergessen werden darf, dass neben den eigentlichen Wirksubstanzen auch verschiedene Cofaktoren, } \\
\text { die bis jetzt nicht genannt wurden, die aber für den geordneten Ablauf des Stoffwechsels benötigt werden, } \\
\text { beigegeben werden sollten, z.B. die Vitamine } B_{2}, B_{6} \text { und } B_{12} \text { sowie Selen und Zink. Außerdem ist auf den } \\
\text { Energiestoffwechsel und den Säure-Basen-Haushalt zu achten. }\end{array}$ & \\
\hline
\end{tabular}

TGF: Transforming growth factor; ADMA: Asymmetrisches Dimethylarginin; eNOS: endotheliale Stickstoffmonoxid-Synthase.

Zur Verbesserung des Stoffwechsels ist unabhängig von den Blutdruckwerten an die Gabe von Vitamin E (gegen Lipidperoxidation) und Vitamin $B_{2}$ (Cofaktor) sowie von Tyrosin und Tryptophan (neuroendokrine Achse) zu denken.

\section{Fazit}

Ernährung, Lebensstil und eine Ergänzung durch gut gemachte Mikronährstoff-
Tab. 2: Initiale Kombinationstherapie mit Mikronährstoffen.

\begin{tabular}{l|l}
\hline Substanz & Dosierung pro Tag \\
\hline Vitamin C & $3 \times 500 \mathrm{mg}$ \\
\hline Vitamin D & $\begin{array}{l}2 \text { Wochen } 10000 \mathrm{IE}(=250 \mathrm{mcg}) / \mathrm{Tag}, \\
\text { dann } 2000 \mathrm{IE} / \mathrm{Tag} \text { unter Laborkontrolle }\end{array}$ \\
\hline Magnesium & $150 \mathrm{mg}$ \\
\hline L-Arginin & $6 \mathrm{~g}$ \\
\hline Omega-3-Fettsäuren & $1,5 \mathrm{~g}$ \\
\hline Resveratrol & $125 \mathrm{mg}$
\end{tabular}




\section{Leitsubstanzen bei Hypertonie}

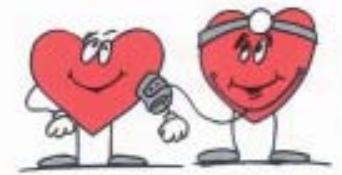

\begin{tabular}{|c|c|c|c|c|c|c|c|c|c|c|c|}
\hline Wirkstoff (evidenzbasiert) & Dosierung & $6 \div$ & 总 & 号 & ᄋ̊ & के के & 造 & एँ & 娄总 & లై & 亏ूँ \\
\hline $\begin{array}{l}\text { Vitamin C } \\
\text { Vitamin E } \\
\text { Polyphenole } \\
\text { (z.B. Resveratrol, Flavonoide) } \\
\text { Coenzym Q } 10\end{array}$ & $\begin{array}{l}500-1500 \mathrm{mg} \\
400-1000 \mathrm{mg} \\
1,5-3 \mathrm{~g} \\
(125 \mathrm{mg} / 50 \mathrm{mg}) \\
100-150 \mathrm{mg}\end{array}$ & $\begin{array}{l}x \\
x \\
x \\
x\end{array}$ & $\begin{array}{l}x \\
X\end{array}$ & $\mathrm{x}$ & $\begin{array}{l}x \\
x \\
x \\
x\end{array}$ & $\mathrm{x}$ & & & $x$ & & $\mathrm{x}$ \\
\hline $\begin{array}{l}\text { Vit. } \mathrm{B}_{1} / \mathrm{B}_{3} \\
\text { Vit. } \mathrm{B}_{2} / \mathrm{B}_{6} \\
\mathrm{~B}_{12} \\
\text { Folsäure } \\
\text { Magnesium } \\
\text { Kalium }\end{array}$ & $\begin{array}{l}40 / 100 \mathrm{mg} \\
40 / 25 \mathrm{mg} \\
0,015 \mathrm{mg} \\
0,4-5 \mathrm{mg} \\
150-400 \mathrm{mg} \\
2-5 \mathrm{~g}\end{array}$ & & & $\begin{array}{l}x \\
x \\
x \\
x \\
x\end{array}$ & $\begin{array}{l}x \\
x \\
x \\
x \\
x\end{array}$ & $\begin{array}{l}x \\
x \\
x \\
x \\
x\end{array}$ & $x$ & $\begin{array}{l}x \\
x\end{array}$ & & & $\begin{array}{l}x \\
x \\
x \\
x\end{array}$ \\
\hline $\begin{array}{l}\text { Ungesättigte Fettsäuren } \\
\text { L-Arginin } \\
\text { L-Tryptophan (oder 5-HTP) } \\
\text { L-Tyrosin }\end{array}$ & $\begin{array}{l}1,5-6 \mathrm{~g} \\
2-6 \mathrm{~g} \\
0,5-3 \mathrm{~g} \\
0,2-3 \mathrm{~g}\end{array}$ & & $\mathrm{x}$ & & $\begin{array}{l}x \\
X\end{array}$ & $\begin{array}{l}x \\
x\end{array}$ & $\mathrm{x}$ & $x$ & $x$ & $\mathrm{x}$ & \\
\hline Vitamin $\mathrm{D}_{3}$ & $25 \mu \mathrm{g}$ und mehr & & $\mathrm{x}$ & & & & & $\mathrm{x}$ & & & \\
\hline
\end{tabular}

\section{Cave: Berücksichtigung von weiteren Cofaktoren (z.B. Selen und Zink), Energiestoffwechsel und Săure-Basen-Haushalt}

\section{Cave: Bei „Diăt" (z.B. Diabetes, Adipositas) Bedarf an Mikronăhrstoffen nicht gedeckt}

Abb. 3: Leitsubstanzen der orthomolekularen Medizin bei Hypertonie. @ U Udo Böhm

medizin haben aus meiner Sicht einen besonders hohen Stellenwert in Prävention und Therapie der Hypertonie. Besonders erfolgversprechend erscheint mir ein Gesamtkonzept, dessen Basis aus einer Optimierung des allgemeinen Lebensstils und der Ernährung besteht, und das - soweit sinnvoll - zunächst durch eine individuelle Kombination dosisangepasster Mikronährstoffe ergänzt wird. Erst wenn damit (auch nach evtl. Veränderung der Mikronährstoffkombination) nach 3 Monaten kein ausrei- chender Erfolg erreicht wird, können die Maßnahmen durch die unter „klassischer“ medikamentöser Therapie genannten Substanzen komplettiert werden.

\section{Servicekasten}

Eine komplette Curriculums-Ausbildung in Orthomolekularmedizin bietet seit über 10 Jahren das Forum orthomolekulare Medizin.

www.f-o-m.de
Interessenkonflikte: Der Autor erklärt, dass keine wirtschaftlichen oder persönlichen Verbindungen bestehen.

Die Studienzusammenfassungen finden Sie im Internet unter:

\section{www.thieme-connect.de/ejournals}

\section{Online zu finden unter:}

http://dx.doi.org//10.1055/s-0033-1357605

\section{Literatur}

[1] Appel LI, Moore TJ et al. A clinical trial of the effects of dietary patterns on blood pressure. N Engl J Med 1997; 336: 1117-1124 [Ib]

[2] Margetts BM, Beilin LJ, Vandongen T et. al. Vegetarian diet in mild hypertension, a randomized controlled trial. BMJ 1986, 293: $1468-1471$

[3] Núñez-Córdoba JM et al. The Mediterranean diet and incidence of hypertension the Seguimiento Universidad de Navarra (SUN) Study. Am J Epidemiol 2009; 169: 339-46

[4] Kokkinos P et al. Dietary influences on blood pressure: the effect of the Mediterranean diet on the prevalence of hypertension. J Clin Hypertens 2005; 7: 165-70 quiz $171-2$
[5] Panagiotakos DB et al. Status and management of hypertension in Greece: role of the adoption of a Mediterranean diet: the Attica study. J Hypertens 2003; 21: 1483-9

[6] Houston MC. The role of cellular micronutrient analysis, nutraceuticals, vitamins, antioxidants and minerals in the prevention and treatment of hypertension and cardiovascular disease; Ther Adv Cardio- 
vasc Dis. 2010; 4: 165-183 und Houston MC. Nutrition and nutraceutical supplements in the treatment of hypertension. Expert Rev Cardiovasc Ther 2010; 8: 82133

[7] Grossman E. Does increased oxidative stress cause hypertension? Diabetes Care 2008; 31 Suppl 2: 185-9

[8] Montezano AC, Touyz RM. Oxidative stress, Noxs, and hypertension: experimental evidence and clinical controversies. Ann Med 2012; 44 Suppl 1: S2-16

[9] Touyz RM, Briones AM. Reactive oxygen species and vascular biology: implications in human hypertension. Hypertens Res 2011; 34: 5-14

[10] Escobales N, Crespo MJ. Oxidative-nitrosative stress in hypertension. Curr Vasc Pharmacol 2005; 3: 231-46

[11] Harrison DG, Gongora MC. Oxidative stress and hypertension. Med Clin North Am 2009; 93: 621-35

[12] Tousoulis D et al. Effects of vitamin C on intracoronary L-arginine dependent coronary vasodilatation in patients with stable angina. Heart 2005; 91: 1319-23

[13] Duffy SJ, Gokce N, Holbrook M et al. Treatment of hypertension with ascorbic acid. The lancet 1999; 9195: 354

[14] Block G et al. Vitamin C in plasma is inversely related to blood pressure and change in blood pressure during the previous year in young black and white women. Nutrition Journal 2008; 7: 35

[15] Lee JH et al. Vitamin D deficiency: An important, common, and easily treatable cardiovascular risk factor? J Am Coll Cardiol 2008; 52: 1949-1956

[16] Wang TJ et al.; Vitamin D deficiency and risk of cardiovascular disease; Circulation 2008; 117: 503-511
[17] Li YC et al. Vitamin D: a negative endocrine regulator of the renin-angiotensin system and blood pressure. J Steroid Biochem Mol Biol 2004; 89-90: 387-92

[18] Vaidya A, Williams JS. The relationship between vitamin $D$ and the renin-angiotensin system in the pathophysiology of hypertension, kidney disease, and diabetes. Metabolism 2012; 61: 450-8

[19] Arora $S$ et al. Thiamine (vitamin $B_{1}$ ) improves endothelium-dependent vasodilatation in the presence of hyperglycemia. Ann Vasc Surg 2006; 20: 653-8

[20] Bays HE, Rader DJ. Does nicotinic acid (niacin) lower blood pressure? Int J Clin Pract 2009; 63: 151-159

[21] Bays HE et al. Blood pressure-lowering effects of extended-release niacin alone and extended-release niacin/laropiprant combination: a post hoc analysis of a 24-week, placebo-controlled trial in dyslipidemic patients. Clin Ther 2009; 31: 115-22

[22] Forman JP et al. Folate intake and the risk of incident hypertension among US women. JAMA 2005; 293: 320-329

[23] Bechir M et al. Folic acid improves baroreceptor sensitivity in hypertension. J Cardiovasc Pharmacol 2005; 45: 44-8

[24] Wu CJ et al. Impact of adding folic acid, vitamin $\mathrm{B}(12)$ and probucol to standard antihypertensive medication on plasma homocysteine and asymmetric dimethylarginine levels of essential hypertension patients; Zhonghua Xin Xue Guan Bing Za Zhi 2012; 40: 1003-8

[25] Broker C et al. Vitamin E prevents extensive lipid peroxidation in patients with hypertension. Br J Biomed Sci 2003; 60: 5-8

[26] Poeggler B. L-Arginin schützt vor nitrosativem Stress: Stickstoffmonoxid als endogener Regulator des nitrosativen Stoffwechsels. Perfusion 2012; 25: 40-434
[27] Facchinetti F et al. L-arginine infusion reduces blood pressure in preeclamptic women through nitric oxide release. J Soc Gynecol Investig 1999; 6: 202-7

[28] Palloshi A et al. Effect of oral L-arginine on blood pressure and symptoms and endothelial function in patients with systemic hypertension, positive exercise tests, and normal coronary arteries. Am J Cardiol 2004; 93: 933-5

[29] Higashi Y et al. Intravenous administration of L-arginine inhibits angiotensin-converting enzyme in humans. J Clin Endocrinol Metab 1995; 80: 2198-202

[30] Burke BE et al. Randomized, double-blind, placebo-controlled trial of coenzyme Q10 in isolated systolic hypertension. South Med J 2001; 94: 1112-1117

[31] Ho MJ et al. Blood pressure lowering efficacy of coenzyme Q10 for primary hypertension. Cochrane Database Syst Rev 2009; 4: CD007435. doi: 10.1002/14651858. CD007435.pub2.

[32] Rosenfeldt FL et al. Coenzyme Q10 in the treatment of hypertension: a meta-analysis of the clinical trials. J Hum Hypertens 2007; 21: 297-306

[33] Digiesi V et al. Effect of coenzyme Q10 on essential arterial hypertension. Current Therapeutic Research 1990; 47: 841-845 und Singh RB et al. Effect of hydrosoluble coenzyme Q10 on blood pressures and insulin resistance in hypertensive patients with coronary artery disease. J Hum Hypertens 1999; 13: 203-208 und Langsjoen $\mathrm{PH}$ et al. Treatment of essential hypertension with Coenzyme Q10. Molec Aspects Med 1994; 15: 265-272

[34] Stolarz-Skrzypek K et al. Sodium and potassium and the pathogenesis of hypertension. Curr Hypertens Rep 2013; 15: 122 30 
[35] van Bommel E, Cleophas T. Potassium treatment for hypertension in patients with high salt intake: a meta-analysis. Int J Clin Pharmacol Ther 2012; 50: 478-82

[36] Cappuccio FP, MacGregor GA. Does potassium supplementation lower blood pressure? A meta-analysis of published trials. J Hypertens 1991; 9: 465-473

[37] Heran BS et al. Blood pressure lowering efficacy of potassium-sparing diuretics (that block the epithelial sodium channel) for primary hypertension. Cochrane Database Syst Rev 2012; 11: CD008167. doi: 10.1002/14651858.CD008167.pub3.

[38] Li S et al. Oral low-dose magnesium gluconate preventing pregnancy induced hypertension; Zhonghua Fu Chan Ke Za Zhi 1997; 32: 613-615

[39] Rudnicki M et al. Comparison of magnesium and methyldopa for the control of blood pressure in pregnancies complicated with hypertension. Gynecol Obstet Invest 2000; 49: 231-235

[40] Sanjuliani AF et al. Effects of magnesium on blood pressure and intracellular ion levels of Brazilian hypertensive patients. Int J Cardiol 1996; 56: 177-183

[41] Witteman JC et al. Reduction of blood pressure with oral magnesium supplementation in women with mild to moderate hypertension. Am J Clin Nutr 1994; 60: 129-135

[42] Wirell MP et al. Nutritional dose of magnesium in hypertensive patients on beta blockers lowers systolic blood pressure: a double-blind, cross-over study. J Intern Med 1994; 236: 189-195

[43] Itoh K et al. The effects of high oral magnesium supplementation on blood pressure, serum lipids and related variables in apparently healthy Japanese subjects. $\mathrm{Br} \mathrm{J}$ Nutr 1997; 78: 737-50

[44] Boenaa KH et al. Effect of eicosapentaenic and docosaheaxaenoic acids on blood pressure in hpertension; a population-base intervention trial from the tromsoe study. $\mathrm{N}$ Engl J Med 1990; 322; 795-801

[45] Cicero AF, Ertek S, Borghi C. Omega-3 polyunsaturated fatty acids: their potential role in blood pressure prevention and management. Curr Vasc Pharmacol. 2009; 7: $330-337$

[46] Albert CM et al. Blood levels of long-chan $n$ 3 fatty acids and the risk of sudden death. $\mathrm{N}$ Engl J Med 2002; 346: 1113-1118

[47] He J et al. Effect of Soybean Protein on Blood Pressure: A Randomized, Controlled Trial. Ann Intern Med 2005; 143: 1-9

[48] Buijsse B et al. Cocoa intake, blood pressure, and cardiovascular mortalitiy. The Zutphen Elderly Study. Arch Intern Med 2006; 166: 411-417

[49] Taubert D et al. Effects of low habitual cocoa intake on blood pressure and bioactive nitric oxide; JAMA 2007; 298: 49-60

[50] Mennen LI et al. Consumption of foods rich in flavonoids is related to a decreased cardiovascular risk in appaently healthy french women. J Nutr 2004; 134: 923-926

[51] Wallerath T et al. Reveratrol, a polyphenolic phytoalexin present in red wine, enhances expression and activity of endothelial nitric oxide synthase. Circulation 2002; 106 : 1652-1658

[52] Wallerath T et al. Red Wine increases the expression of human endothelial nitric oxide synthase. J Am Coll Cardiol 2003; 41: 471-478

[53] Lekakis J et al. Polyphenolic compounds from red grapes acutualy improve endothelial function in patients with coronary heart disease. Eur J Cardiovasc Prev Rehabil 2005; 12: 596-600

[54] Wong RHX et al. Acute effects of Resveratrol-supplementation on cardiovascular risk factors. Nutrition Society of Australia and Nutrition Society of New Zealand 2009 Joint Annual Scientific Meeting. 6 November 2009 und Wong RHX et al. Acute resveratrol supplementation improves flow-mediated dilatation in overweight/obese individuals with mildly elevated blood pressure. Nutrition Metabolism and Cardiovascular Diseases 2010 (in press).

[55] Corder R, Douthwaite JA, Lees DM et al. Endothelin-1 synthesis reduced by red wine. Nature 2001; 414; 863-864

[56] Carrizzo A et al. Resveratrol improves vascular function in patients with hypertension and dyslipidemia by modulating NO metabolism. Hypertension 2013; 62: 35966

[57] Moreno-Luna R et al. Olive oil polyphenols decrease blood pressure and improve endothelial function in young women with mild hypertension. Am J Hypertens 2012; 25: 1299-304

[58] Ferrara LA et al. Olive oil and reduced need for antihypertensive medications. Arch Intern Med 2000; 160: 837-42

[59] Galleano M et al.Hypertension, nitric oxide, oxidants, and dietary plant polyphenols. Curr Pharm Biotechnol 2010; 11: 837-48

[60] Antonello M et al. Prevention of hypertension, cardiovascular damage and endothelial dysfunction with green tea extracts. Am J Hypertens 2007; 20: 1321-8

[61] Yang YC et al. The protective effect of habitual tea consumption on hypertension. Arch Intern Med 2004; 164: 1534-40

[62] McKay DL. Hibiscus sabdariffa L. Tea (Tisane) lowers blood pressure in prehypertensive and mildly hypertensive adults. American Heart Association 2008 Scientific Sessions, Abstract 3278, November 10 , 2008. Circulation 2008; 118: 1123

[63] Perez-Vizcaino F et al. Antihypertensive effects of the flavonoid quercetin. Pharmacol Rep 2009; 61: 67-75

[64] Steinberg FM et al. Soy protein with isoflavones has fevorable effects on endothelial function that are indipendent of lipid and antioxidant effects in healthy postmenopausale women. Am J Clin Nutr 2003; 8: $123-130$

[65] Lim U et al. Homocysteine and Blood Pressure in the Third National Health and Nutrition Examination Survey, 1988-1994. Am J Epidemiol 2002; 156: 1105-1113

[66] Sawai A et al. Influence of mental stress on the plasma homocysteine level and blood pressure change in young men. Clin Exp Hypertens 2008; 30: 233-41

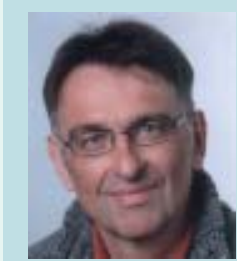

Udo Böhm, Jahrgang 1951, von 1982-2009 in Unterwössen/Oberbayern niedergelassen. Vortragstätigkeit, Konzeptentwicklung und Veröffentlichungen in den Bereichen Gesundheitsmedizin und Praxismanagement; Mitbegründer \& Vorstandsmitglied des Forum Orthomolekulare Medizin; Lehrbeauftragter für Allgemeinmedizin der LMU München; Vorstandsmitglied der Deutschen Gesellschaft für präventive Medizin.

\section{Korrespondenzadresse}

\section{Dr. med. Udo Böhm}

Kruchenhausen 35

83246 Unterwössen

E-Mail: agg-uw@kabelmail.de

\section{Zum Weiterlesen}

Böhm U, Muss C, Hrsg. Rationelle Therapie in der Mikronährstoffmedizin. Bremen: UNI-MED; 2011 Böhm U, Muss C, Pfisterer M. Rationelle Diagnostik in der Orthomolekularen Medizin. E-Book

[67] Fakhrzadeh $\mathrm{H}$ et al. Plasma homocysteine concentration and blood pressure in healthy Iranian adults: the Tehran homcysteine survey (2003-2004). J Hum Hypertens 2005; 19: 869-76

[68] Dinavahi R et al. Plasma homocysteine concentration and blood pressure in young adult African Americans. Am J Hypertens 2003; 16: 767-70

[69] van Guldener C et al. Homocysteine and blood pressure. Curr Hypertens Rep 2003; 5: 26-31

[70] Tayama J et al. Higher plasma homocysteine concentration is associated with more advanced systemic arterial stiffness and greater blood pressure response to stress in hypertensive patients. Hypertens Res 2006; 29: 403-9

[71] Stehouwer CD, van Guldener C. Does homocysteine cause hypertension? Clin Chem Lab Med 2003; 41: 1408-11

[72] Forman JP et al. Folate intake and the risk of incident hypertension among US women. JAMA 2005; 293: 320-9 


\section{Studienzusammenfassungen}

1. Appel LI, Moore TJ et al. 1997:

Eine Ernährung reich an Früchten, Gemüse und fettarmen Produkten mit reduziertem Gesamtfett und reduzierten gesättigten Fettsäuren kann den Blutdruck erniedrigen (bei Hypertonikern um 11,4 mmHg systolisch bzw. 5,5 mmHg diastolisch gegenüber amerikanischer Normalkost) und ist geeignet für Prävention und Therapie von Hypertonie. Randomisiert; 459 Teilnehmer.

\section{Margetts BM, Beilin LJ et al. 1986:} Vegetarische Diät senkt bei Hypertonikern den systolischen Blutdruck um $5 \mathrm{mmHg}$. Randomisierte Cross-over-Studie mit 58 Teilnehmern über 6 Wochen.

\section{Núñez-Córdoba JM et al. 2009:}

Mediterrane Diät reduziert systolischen und diastolischen Blutdruck. Spanish prospective cohort study; 9408 Teilnehmer über 6 Jahre.

\section{Kokkinos P et al. 2005:}

In einem Review wird das Wissen über die Einflüsse der Ernährung auf den Blutdruck dargestellt.

\section{Panagiotakos DB et al. 2003:}

In der griechischen Studiengruppe war die Hypertonieprävalenz bei Männern 38,2\% und bei Frauen 23,9\%. Nur $15 \%$ der Hypertoniker haben einen gut eingestellten Blutdruck. Mediterrane Kost reduziert den Blutdruck. Sie reduziert das Hypertonierisiko um 26\% und erhöht die Chancen für eine normale Blutdruckeinstellung um 36\%; 2282 Teilnehmer.

\section{Houston MC. 2010:}

Mikronährstoffdefizit ist häufig in der Bevölkerung und besonders bei Hypertonikern und Herzkranken aus Gründen der Genetik, des Lebensstils und der Nutzung von Medikamenten. Es findet sich ein signifikanter Unterschied bei zahlreichen intrazellulären Nutrienten und beim Oxidationsstatus zwischen Hypertonikern und Menschen mit normalem Druck. Ersatz des Defizits in Kombination mit ausgewählten hochdosierten Mikronährstoffen und optimierter Ernährung, Bewegung, Gewichtsmanagement, Verzicht auf Rauchen, Reduzierung von Alkohol und Koffein resultiert in einer Kontrolle des Blutdrucks auf einem Ziellevel von $62 \%$ der Hypertoniker über einen Zeitraum von 6 Monaten unter Reduzierung einer Antihypertonikatherapie. Endotheliale Aktivierung, oxidativer Stress, Inflammation und Dysfunktion von glatten Gefäßmuskelzellen sind initiale Ereignisse, die Hypertonie auslösen.

\section{Grossman E. 2008:}

Hypertonie ist mit erhöhtem vaskulärem oxidativem Stress verbunden. Die Frage ist, ob oxidativer Stress eine Ursache oder eine Folge von Hypertonie ist. oxidativer Stress fördert die Proliferation und Hypertrophie von glatten Gefäßmuskelzellen (und Kollagenablagerungen), was die Dicke der Gefäßmedia erhöht und das Gefäßlumen verengt. Er schädigt das Endothelium und die endothelabhängige Gefäßdilatation und erhöht die kontraktile Gefäßaktivität. So kann oxidativer Stress Hypertonie verursachen. Antioxidantientherapie zeigt keinen konsistenten Benefit. Da Antihypertonikatherapie oxidativen Stress reduziert, scheint oxidativer Stress nicht eine Ursache, sondern eine Folge von Hypertonie zu sein.

\section{Montezano AC, Touyz RM. 2012:}

Oxidativer Stress ist nicht der einzige Grund für Hypertonie, aber er verstärkt die Blutdruckerhöhung bei Anwesenheit anderer prohypertensiver Faktoren (z.B. Salz, RAAS-System, sympathische Überaktivität). Eine Hauptursache für oxidativen Stress ist eine Familie nicht phagozytischer NADPH-Oxidasen (Nox 1, 2, 4, 5). Andere Ursachen beinhalten mitochondriale Elektronentransportenzyme, Xanthinoxdase und entkoppelte NOS. Es ist heute klar, dass oxidativer Stress eine wichtige Rolle bei den molekularen Mechanismen spielt, die mit kardiovaskulären und renalen Stö- rungen bei Hypertonie verbunden sind, und dass Hypertonie zu oxidativem Stress führen kann. Review.

\section{Touyz RM, Briones AM. 2011:}

Oxidativer Stress ist Ursache und Folge von Hypertonie. Bei oxidativem Stress handelt es sich um ein Multisystemproblem, das Herz, Nieren, Nervensystem, Gefäße und evtl. das Immunsystem einbezieht. Eine Hauptquelle für ROS ist eine Familie nicht phagozytischer NADPH-Oxidasen (Nox 1, $2,4,5)$. Nox-abhängige ROS sind wichtig für die Regulation der Endothelfunktion und des Gefäßtonus. Oxidativer Stress ist involviert in Endotheldysfunktion, Entzündung, Hypertrophie, Apoptose, Migration, Fibrose, Angiogenese und Verdünnung, wichtige Prozesse im Gefäßremodelling bei Hypertonie. Review.

\section{Escobales N, Crespo MJ. 2005:}

ROS können zu Gefäßabnormitäten beitragen, die zu Hypertonie führen. Es gibt Evidenz, dass ROS im Gefäßsystem hauptsächlich durch NADPH-Oxidasen in einem Angiotensin-II-abhängigen Mechanismus gebildet werden. Aktivierung dieses Enzyms führt zur Produktion von Superoxid und entkoppelt die eNOS, was wiederum oxidativen Stress und eine Erhöhung des gewebsschädigenden Peroxynitrit verursacht. Das kann zu Gefäßdysfunktion führen. Dies kann Gefäßschäden bedingen, Entzündungsförderung, extrazellulärer Matrixreorganisation und Wachstum von glatten Gefäßmuskelzellen führen. Der ROS-Effekt wird vermittelt durch redoxsensitive Stoffe wie Tyrosinkinasen und Phosphatasen, mitogen-aktivierte Proteinkinasen, Transkriptionsfaktoren, MatrixMetalloproteinasen, Peroxisomproliferator-aktivitierten Rezeptor-alpha, Poly (ADP-Ribose) Polymerase-1, Ca(2+)-Signalmechanisemn und weitere Faktoren wie Cyclophilin A und Heat Shock Protein-90alpha. Diese Redox-sensitiven Stoffe scheinen eine Rolle für normale Gefäßfunktion zu spielen. 
11. Harrison DG, Gongora MC. 2009:

Hypertensive Stimuli wie Salz und Angiotensin II fördern die ROS-Produktion in Gehirn, Niere und Gefäßsystem und diese Stoffe tragen zu Hypertonieentwicklung bei. Die NADH-Oxidase (z.B. durch Angiotensin II gefördert) in diesen Organen ist Hauptquelle für ROS, aber andere Enzyme tragen ebenfalls zur Bildung von ROS bei. Sympathische Nervenendigungen und Angiotensin II aktivieren T-Zellen und die Expression von Signalstoffen, die T-Zellen in die genannten Organe transportieren. T-Zellen aktivieren Zytokine, die dort NADPH-Oxidasen stimulieren, welche Salzretention und Gefäßkonstriktion promoten. Review.

\section{Tousoulis D et al. 2005:}

Vitamin C (parenteral) hat günstige Effekte bei der durch Infusion von Arginin induzierten NO-Verfügbarkeit.

13. Duffy SJ, Gokce N, Holbrook M et al. 1999:

Der Blutdruck von Bluthochdruckpatienten kann durch eine regelmäßige, über einen Monat dauernde Einnahme von Vitamin C gesenkt werden. Randomisierte, doppelblinde, placebokontrollierte Studie.

14. Block G et al. 2008:

Systolischer und diastolischer Blutdruck sind bei den Frauen mit den höchsten Vitamin-C-Spiegeln 4,66 mmHg bzw. 6,04 mmHg niedriger als bei den Frauen mit den niedrigsten Spiegeln. Vitamin $C$ beeinflusst den Blutdruck bei jungen Erwachsenen positiv. National Heart, Lung and Blood Institute Growth and Health Study (Kohortenstudie); 242 Teilnehmer über 10 Jahre.

\section{Lee JH et al. 2008:}

Menschen mit 25-OH-Vitamin D unter $15 \mathrm{ng} / \mathrm{ml}$ haben ein doppelt so hohes 5-Jahres-Risiko für Herzinfarkt, Schlaganfall u.a. kardiovaskuläre Ereignisse wie Menschen mit höheren Werten. Vitamin-D-Defizit ist weit verbreitet und findet sich bei 30$50 \%$ der Bevölkerung. Es aktiviert das Renin-Angiotensin-Aldosteron-System (mit der Folge von Hypertonie und linksventrikulärer Hypertrophie). Außerdem führt es zu einem Anstieg der Parathormonausschüttung (führt zu Insulinresistenz, welche verknüpft ist mit Diabetes, Hypertonie, Entzündung und erhöhtem kardiovaskulärem Risiko). Bei Herzpatienten ist deshalb auf ausreichend hohe Vitamin-D-Spiegel zu achten.

16. Wang TJ et al. 2008:

$37 \%$ der Untersuchten hatten Vitamin-DSpiegel unter $15 \mathrm{ng} / \mathrm{ml}$. Sie hatten durchschnittlich ein erhöhtes kardiovaskuläres Risiko (HR 1,62; 95\%-KI) gegenüber den Teilnehmern mit höheren Werten. Bei Hypertonikern war das Risiko besonders hoch (HR 2,13; 95\%-KI) FraminghamOffspring-Studie; 1739 Teilnehmer.

\section{Li YC et al. 2004:}

Es findet sich eine Korrelation zwischen Vitamin-D-Mangel und Hypertonie. Vitamin D ist ein potenter endokriner Suppressor der Renin-Biosynthese im RAASSystem. Der Mechanismus ist unabhängig von Calcium-Stoffwechsel, Volumen, Salzgehalt und ACE-Feedback-Regulation.

\section{Vaidya A, Williams JS. 2012:}

Vitamin D kann das Renin-System über Interaktion mit dem Vitamin-D-Rezeptor negativ regulieren. Review.

\section{Arora S et al. 2006:}

Vitamin $\mathrm{B}_{1} /$ Thiamin $100 \mathrm{mg}$ i.v. erhöht bei Hyperglykämie die endothelabhängige Vasodilatation der Armarterie.

\section{Bays HE, Rader DJ. 2009:}

Niacin (nur als Nikotinsäure) reduziert den Blutdruck. Es hat einen vasodilatatorischen Effekt und kann zu akuter Hypotonie führen (kann auch Flush verursachen), aber auch langfristig dosisabhängig den Blutdruck senken (durch Dilatation der dermalen Gefäße über eine Aktivierung des DP1-Rezeptors durch PGD2). Review.

\section{Bays HE et al. 2009:}

Niacin reduziert bei Patienten mit Fettstoffwechselstörung signifikant systolischen und diastolischen Blutdruck (zwischen 2,2 und 3,1 mmHg). 1613 Teilnehmer über 24 Wochen.

22. Forman JP et al. 2005:

In der prospektiven Nurses Health Study I mit 93803 Krankenschwestern über 8 Jahre reduzierte eine Folsäurezufuhr von über 1000 mcg gegenüber einer Zufuhr von unter 200 mcg signifikant die Hypertonierate um $46 \%$ (6,7 statt 14,8 Fälle, d.h. RR = 0,54 bzw. eine absolute Risikoreduktion 8 Fälle/ 1000 Personenjahre) bei Frauen von 27-44 Jahren. Bei Frauen von 42-70 Jahren waren es 34,7 statt 40,4 Fälle/1000 Beobachtungsjahre (Folsäure kann die Endothelfunktion verbessern)

\section{Bechir M et al. 2005:}

Die Barorezeptor-vermittelte Modulation der Herzfrequenz ist bei Hypertonie gestört wegen verminderter vagaler Kontrolle. ROS reduzieren die Barorezeptorsensitivität. Folsäure $5 \mathrm{mg}$ verbessert bei Hypertonie signifikant die kardiale und vaskuläre Barorezeptorsensitivität gegenüber Placebo. Placebokontrolliert; 21 Teilnehmer.

\section{Wu CJ et al. 2012:}

Supplementation von Folsäure, $\mathrm{B}_{12}$ und Probucol verbessert bei Hypertonie die endotheliale Funktion, sie reduziert Plasmahomoocystein und ADMA. 120 Teilnehmer über 12 Wochen.

\section{Broker C et al. 2003:}

Hypertoniker haben ein erhöhtes Risiko für LDL-Oxidation. Vitamin E 400 IE reduziert das Risiko. 47 Teilnehmer über 2 Monate.

\section{Poeggler B. 1999:}

L-Arginin ist das Substrat der NO-Synthasen im Endothel der Gefäße. Über die Synthese und anschließende Diffusion von NO in das benachbarte Gewebe wird eine Relaxation der Gefäßmuskulatur erreicht. Der lokale Blutdruck sinkt, da die Gefäßwand die pulsatilen Druckspitzen des Blutstroms bei geringerem Tonus leichter kompensieren kann und sich der Gefäßquerschnitt erhöht. In diesem Zusammenhang ist L-Arginin als NO-Donor mit antihypertensiven Eigenschaften zu betrachten, wenn ein entsprechender Mangel vorliegt. 


\section{Facchinetti F et al. 1999:}

L-Arginin-Infusion $30 \mathrm{~g}$ senkt signifikant den Blutdruck bei Schwangeren, stärker bei Frauen mit Präeklampsie (mit reduzierter NO-Produktion). Es kommt zu verstärkter NO-Produktion und Hypotension. Präeklampsie ist charakterisiert durch Störung des L-Arginin-NO-Wegs.

28. Palloshi A et al. 2004:

L-Arginin $3 \times 2 \mathrm{~g}$ verbessert signifikant Angina pectoris, Blutdruck und Lebensqualität.

\section{Higashi Y et al. 1995:}

L-Arginin-Infusion reduziert Blutdruck, Nierengefäßwiderstand sowie ACE-Aktivität und Angiotensin-II-Spiegel. Sie erhöht die Herzrate und den renalen Plasmaflow. Ein Teil der Wirkung lässt sich also über Inhibierung des Reninsystems erklären.

\section{Burke BE et al. 2001:}

Bei Respondern Senkung RR systolisch um 25,9 mmHg durch Coenzym Q10 2×60 mg. Randomisierte, doppelblinde, placebokontrollierte Studie.

\section{Ho MJ et al. 2009:}

Coenzym Q10 senkt im Durchschnitt den systolischen Blutdruck um $11 \mathrm{mmHg}$ und den diastolischen um $7 \mathrm{mmHg}$. Es ist jedoch wegen der Nichtvergleichbarkeit der eingeschlossenen Studien nicht klar, ob Coenzym Q10 den Blutdruck bei der Langzeitbetreuung von essenzieller Hypertonie senkt. 3 Studien mit 96 Teilnehmern.

\section{Rosenfeldt FL et al. 2007:}

Coenzym Q10 reduziert bei Hypertonikern den systolischen Blutdruck um bis zu $17 \mathrm{mmHg}$ und den diastolischen bis zu $10 \mathrm{mmHg}$ ohne signifikante Nebenwirkungen. Review aus 12 Studien mit 362 Teilnehmern.

33. Digiesi V et al. 1990; Singh RB et al. 1999; Langsjoen PH et al. 1994:

Coenzym Q10 (60 mg 2 × tgl.) senkt bei isolierter systolischer Blutdurckerhöhung den systolischen Blutdruck um durchschnittlich 17,8 mmHg. Es gab keine orthostatischen Blutdruckänderungen. Randomisiert, dop- pelblind, placebokontrolliert; 83 Teilnehmer über 12 Wochen.

\section{Stolarz-Skrzypek K et al. 2013:}

Hoher Salzkonsum erhöht den Blutdruck. Kaliumsubstitution reduziert den Blutdruck. Trotzdem sollte gegenwärtig keine generalisierte Salzreduktion oder eine generelle Kaliumsubstitution bei Hypertonikern empfohlen werden Eine Ernährung reich an Obst und Gemüse ist empfehlenswert.

35. van Bommel E, Cleophas T. 2012: Kaliumsupplementierung reduziert den Blutdruck bei Hypertonikern signifikant systolisch um 7,1 mmHg und diastolisch um 4,9 mmHg (v.a. bei salzreicher Ernährung). Patienten mit reduzierter Salzaufnahme profitieren weniger von Kalium. Metaanalyse aus 10 Studien.

36. Cappuccio FP, MacGregor GA. 1991: Kaliumsupplementation oral reduziert signifikant systolischen $(5,9 \mathrm{mmHg})$ und diastolischen $(3,4 \mathrm{mmHg})$ Blutdruck. Die Blutdrucksenkung ist höher bei Patienten mit hohem Blutdruck $(8,2 \mathrm{mmHg}$ und $4,5 \mathrm{mmHg}$ ) und bleibt stabil bei längerer Behandlungszeit. Review aus 19 Studien mit 586 Teilnehmern.

\section{Heran Bs et al. 2012:}

Kaliumsparende Diuretika (Amilorid, Triamteren) haben in niedriger Dosierung und in Kombination mit anderen Medikamenten keinen signifikanten Blutdrucksenkenden Effekt. Es gibt keine Studien mit hohen Dosierungen.

\section{Li S et al. 1997:}

Hypertonie in der Schwangerschaft: Magnesiumglukonat $3 \mathrm{~g} /$ Tag reduziert gegenüber Placebo effektiv die Entwicklung einer Schwangerschaftshypertonie von $16 \%$ auf $4 \%$. Randomisierte, doppelblinde, placebokontrollierte Studie; 131 Schwangere.

\section{Rudnicki M et al. 2000:}

Hypertonie in der Schwangerschaft: Frauen mit schwangerschaftsbedingter Hypertonie erhielten entweder Magnesium oder Methyldopa (250 mg $4 \times$ Tag). Die Gruppe mit Magnesium hatte signifikant geringere systolische und diastolische Blutdruckwerte als die mit Methyldopa. Randomisierte, kontrollierte klinische Studie; 33 Teilnehmer.

\section{Sanjuliani AF et al. 1996:}

Magnesium 600 mg/Tag reduziert bei Respondern ( $40 \%$ der Studienteilnehmer) signifikant systolischen, diastolischen und mittleren Blutdruck (>10 mmHg) sowie intrazelluläres Natrium bei milder bis mittelschwerer Hypertonie. Randomisierte, doppelblinde, placebokontrollierte CrossOver-Studie.

\section{Witteman JC et al. 1994:}

Magnesiumaspartat (20 mmol $\mathrm{Mg} / \mathrm{Tag}$ ) senkt systolischen um 2,7 mmHg (95\%KI) und diastolischen Blutdruck um 3,4 mmHg mehr als Placebo bei milder bis mittelschwerer (unbehandelter) Hypertonie. Randomisierte, doppelblinde, placebokontrollierte Studie; 6 Monate, 91 Teilnehmer.

42. Wirell MP et al. 1994:

Magnesiumaspartat (365 mg Mg/Tag) zusätzlich zu einer unveränderten Beta-Blocker-Therapie nach einer Placeboperiode gegeben senkt signifikant den systolischen Blutdruck im Liegen und Stehen. Randomisierte, doppelblinde Cross-over-Studie über 8 Wochen.

\section{Itoh K et al. 1997:}

Hypertonie und Fettstoffwechsel: Magnesium $(\mathrm{Mg}(\mathrm{OH}) 2$; 411-548 mg Mg/Tag) senkt signifikant systolischen und diastolischen Blutdruck. Lecithin-Cholesterin-Acyltransferase; HDL und Apolipoprotein AI steigen signifikant an und das Verhältnis GesamtCholesterin zu HDL nimmt signifikant ab. Randomisierte, doppelblinde, placebokontrollierte Studie.

\section{Boenaa KH et al. 1990:}

Fischöl $6 \mathrm{~g}$ reduziert bei Hypertonikern signifikant den systolischen Druck um 4,6 mmHg und den diastolischen Druck um $3 \mathrm{mmHg}$ im Vergleich zu Placebo. Randomisiert; 156 Teilnehmer über 10 Wochen. 
45. Cicero AF, Ertek S, Borghi C. 2009:

Omega-3-Fettsäuren verbessern leicht aber nicht signifikant arterielle Hypertonie. Sie regulieren vasomotorischen Tonus, renale Salzexkretion, reduzieren Vasokonstriktorenproduktion, ACE-Aktivität, Bildung von Angiotensin II und TGF- $\beta$-Expression, erhöhen NO-Bildung und aktivieren den Parasympathikus. Es ergibt sich eine verbesserte Vasodilatation.

\section{Albert CM et al. 2002:}

Die Blutspiegel von Omega-3-Fettsäuren sind streng signifikant assoziiert mit einem reduzierten Risiko für plötzlichen Tod bei Männern mit KHK (RR 0,19; 95\%-KI für höchste gegen niedrigste Spiegel). Prospektive Physicians Health Study; 278 Teilnehmer über 17 Jahre.

\section{He J et al. 2005:}

Sojabohnenprotein $40 \mathrm{~g} /$ Tag senkt hochsignifikant Blutdruck durchschnittlich systolisch um 4,31 mmHg und diastolisch um 2,76 $\mathrm{mmHg}$ und bei Hypertonikern um 7,88 mmHg bzw. um 5,27 mmHg im Vergleich zu Placebo. Randomisiert, doppelblind, kontrolliert; 302 Teilnehmer über 12 Wochen.

48. Buijsse B et al. 2006:

Bei Männern mit dem höchsten Kakaokonsum (mit Polyphenolen) ist der systolische Blutdruck um 3,7 mmHg und der diastolische Blutdruck um 2,1 mmHg signifikant niedriger als bei denjenigen mit dem geringsten Kakaokonsum. Die Männer mit dem höchsten Kakaokonsum haben außerdem ein um 50\% signifikant niedrigeres Risiko an Herz-Kreislauf-Erkrankungen zu sterben. Prospektive Kohortenstudie; 470 Teilnehmer über 15 Jahre.

49. Taubert D et al. 2007:

Dunkle polyphenolreiche Schokolade senkt signifikant den systolischen Blutdruck um 2,9 mmHg und den diastolischen Druck um 1,9 mmHg gegenüber Placebo Die Prävalenz von Hypertonie verringert sich um $21 \%$ (von $86 \%$ auf $68 \%$ ). Außerdem kommt es zu einem kontinuierlichen Anstieg der Plasma-S-Nitrosoglutathion-Spiegel (wichtigste Transportform des bioaktiven Stick- stoffmonoxids) um 0,23 nmol/l. Randomisiert, doppelblind, placebokontrolliert; 44 Teilnehmer.

\section{Mennen LI et al. 2004:}

Hohe Flavonoidaufnahme ist verbunden mit erniedrigtem Blutdruck und reduziertem kardioaskulären Risiko bei Frauen (OR 0,32; 95\%-KI). SU.VI.MAX Study; 2291 Teilnehmer über 8 Jahre.

\section{Wallerath T et al. 2002:}

Resveratrol upreguliert die Expression und stabilisiert die eNOS mRNA in menschlichen Nabelschnurendothel-Zellen zeitund konzentrationsabhängig (bis zum 2,8Fachen) sowie die Expression des eNOSProteins und die NOS-abhängige NO-Produktion.

\section{Wallerath T et al. 2003:}

Es wurden deutsche und französische Rotweine hinsichtlich einer Steigerung der eNOS-Aktivität (führt zur Freisetzung des vasodilatierenden NO) verglichen. Die französischen Weine waren weitaus potenter als die Deutschen. Alkohol alleine hatte kaum einen Effekt. Wahrscheinlich kommt es auf den Gehalt an sekundären Pflanzenstoffen an. Die Inkubation von Endothelzellen mit Rotwein aus Frankreich upreguliert die Expression der eNOSmRNA und des eNOS-Proteins. Im Gegensatz dazu zeigten Rotweine aus Deutschland keine oder nur geringe Auswirkungen auf die eNOS-Expression. Kein signifikanter Unterschied in der eNOSmRNA-Expression konnte bei Rotweinen aus Frankreich zwischen „Barrique“ und „Nicht-Barrique“ gefunden werden. Mit französischem Rotwein behandelte Zellen produzieren bis zu $3 \times$ mehr bioaktive NO als die Kontrollen. Französische Rotweine erhöhen die Aktivität der eNOS-Promotoren und die eNOSmRNA Stabilität.

\section{Lekakis J et al. 2005:}

Polyphenole (600 mg) inkl. 0,9 mg TransResveratrol aus roten Trauben verbessern signifikant und akut die Endothelfunktion und Vasodilatation bei Patienten mit KHK. Randomisiert, placebokontrolliert; 30 Teilnehmer.
54. Wong RHX et al. 2009:

Wong RHX et al. 2010; Resveratrol verbessert bei Übergewichtigen und Hypertonikern dosisabhängig die Vasodilatation. Doppelblinde, randomisierte Cross-overStudie; 19 Teilnehmer.

55. Corder R, Douthwaite JA, Lees DM et al. 2001:

Resveratrol und andere Polyphenole im Rotwein hemmen effektiv einen für die KHK wichtigen pathogenetischen Faktor, den (starken) Vasokonstriktor Endothelin 1. Weißwein und Rose haben nicht genügend Polyphenole für diesen Effekt.

\section{Martin et al. 2001:}

Resveratrol fördert Gefäßrelaxation und verbessert endotheliale Dysfunktion über eine Modulation des NO-Metabolismus (1. über einen 5‘-Adenosinmonophosphat aktivierten Proteinkinase vermittelten Anstieg der eNOS-Aktivität, 2. über einen Anstieg der Tetrahydrobiopterinspiegel, welche ebenfalls die eNOS-Aktivität erhöhen, 3. über eine Reduzierung von oxidativem Stress durch Überexpression der mSOD).

\section{Carrizzo A et al. 2013:}

Eine Ernährung mit viel polyphenolreichem Olivenöl (ca. 30 mg/Tag) kann den Blutdruck signifikant reduzieren (systolisch um 7,91 mmHg und diastolisch um 6,65 mmHg). Auch ADMA, oxLDL und CRP wurden verringert, die Endothelfunktion wurde verbessert. Doppelblind, randomisiert; 24 Teilnehmer über 4 Monate.

\section{Moreno-Luna R et al. 2012:}

Eine Reduktion gesättigter Fettsäuren verbunden mit der Zufuhr von Extra vergine Olivenöl (mit MUFA) reduziert signifikant die Dosis der antihypertensiven Therapie um $48 \%$ (evtl. durch Polyphenol-stimulierte erhöhte NO-Spiegel). 8 Teilnehmer benötigten keine antihypertensive Therapie am Ende der Studie. Doppelblind, randomisiert; 23 Teilnehmer über $2 \times 6$ Monate.

\section{Ferrara LA et al. 2000:}

Polyphenole beeinflussen die Endothelfunktion (über Regulierung der NO-Biover- 
fügbarkeit) und als Konsequenz auch den Blutdruck. Review.

60. Galleano M et al. 2010:

Grüntee-Extrakt verhindert bei Ratten Hypertonie und Organschäden infolge hoher Angiotensin-II-Spiegel und reduziert Superoxidanionbildung.

\section{Antonello M et al. 2007:}

Grüntee-Zufuhr (120 ml/Tag oder mehr über $1 \mathrm{Jahr}$ ) reduziert signifikant das Hypertonierisiko bei Chinesen (um $46 \%$ bei $120-599 \mathrm{ml} /$ Tag und um $65 \%$ bei mehr als $599 \mathrm{ml} /$ Tag). 1507 Teilnehmer.

\section{Yang YC et al. 2004:}

Hibiskustee 3 Tassen tgl. (enthält Anthocyane, Flavone, Flavonole und Phenolsäure „mit antioxidativer, antiatherosklerotischer und ACE-Hemmer-ähnlicher Wirkung“) senkt den Blutdruck signifikant systolisch um 7-14 mmHg und diastolisch um $3 \mathrm{mmHg}$. Diese RR-Senkung resultiert in einer Reduktion der Schlaganfallsterblichkeit um $8 \%$, der KHK-Mortalität um 5\% und der Gesamtmortalität um 4\%. Randomisiert, doppelblind, placebokontrolliert; 65 Teilnehmer über 6 Wochen.

63. McKay DL. 2008:

Das Flavonoid Quercetin reduziert dosisabhängig den Blutdruck. Dieser Effekt kann zur Reduzierung des Risikos für Herzinfarkt und Schlaganfall beitragen. Review.

\section{Perez-Vizcaino F et al. 2009:}

Soja-Proteinzufuhr hat bei postmenopausalen Frauen signifikate positive vaskuläre Effekte (Vasodilatation) unabhängig von ihren Wirkungen auf Lipide und Radikale. Randomisiert, doppelblind; 28 Teilnehmer.
65. Steinberg FM et al. 2003:

Homocystein hat eine unabhängige positive Verknüpfung mit hohem Blutdruck. Eine Erhöhung des Homocysteins um $5 \mathrm{mcmol} / \mathrm{l}$ führt zu einer Erhöhung des systolischen und diastolischen Blutdrucks um 1,2 bzw. 0,7 mmHg bei Frauen. Frauen haben bei den höchsten im Vergleich zu den niedrigsten Homocysteinspiegeln ein 3-fach erhöhtes Hypertonierisiko, Männer ein 2-fach erhöhtes Hypertonierisiko (95\%-KI).

\section{Lim U et al. 2002:}

Studenten mit Hypertonie bei den Eltern haben einen signifikant erhöhten Homocysteinspiegel. Mentaler Stress erhöht signifikant Herzfrequenz, Blutdruck, Sympathikusaktivität und Homocystein. Homocystein korreliert signifikant mit der Änderung des systolischen Blutdrucks und der sympathovagalen Balance. 27 Teilnehmer.

\section{Sawai A et al. 2008:}

Bei hypertensiven Patienten ist der Homocysteinspiegel höher, aber signifikant nur bei Männern. Vitamin $B_{12}$ hat entgegengesetzte Effekte zu Homocystein und dient als Schutzfaktor. Cardiovascular Risk Factors Survey (Monica-Project) mit 1214 Teilnehmern.

\section{Fakhrzadeh H et al. 2005:}

Homocystein ist bei Hypertonikern höher als bei Blutdruckgesunden. 175 Teilnehmer.

\section{Dinavahi R et al. 2003:}

Homocysteisenkende Therapie mit Folsäure und Vitamin $\mathrm{B}_{12}$ reduziert den Blutdruck. Homocystein kann die arterielle Steifigkeit erhöhen und zu Endotheldysfunktion, erniedrigter NO-Verfügbarkeit, niedrigem Folsäurestatus und Insulinresistenz beitra- gen. Man weiß aber derzeit nicht genau, ob der Blutdruck durch die Vitamine oder die Homocysteinsenkung reduziert wird.

70. van Guldener C et al. 2003:

Die Pulswellengeschwindigkeit korreliert mit dem Homocysteinspiegel und dem Blutdruck. Bei Teilnehmern mit hohem Homocystein war insbesondere der systolische Blutdruck unter Stress (als Zeichen der erhöhten Arteriensteifigkeit) höher als bei denen mit normalem Homocystein. 50 Teilnehmer.

\section{Tayama J et al. 2006:}

In einer sorgfältig durchgeführten Studie war jeder $5 \mathrm{mcmol} / \mathrm{l}$ Anstieg von Homocystein mit einem Anstieg des systolischen bzw. diastolischen Blutdrucks um 0,7/0,5 mmHg bei Männern und um 1,2/ $0,7 \mathrm{mmHg}$ bei Frauen verbunden, unabhängig von Nierenfunktion und Vitamin-B-Status. Eine homocysteinsenkende Therapie reduziert auch den Blutdruck. Begründet wird das damit, dass Homocystein Arterienkonstriktion, renale Dysfunktion, erhöhte Arteriensteifigkeit und erhöhte Natriumrückresorption bedingt. Man weiß aber noch nicht sicher, ob die Blutdrucksenkung durch die Vitamine oder durch Homocysteinsenkung erreicht wird.

72. Stehouwer CD, van Guldener C. 2003: Höhere Folataufnahme (ab $1 \mathrm{mg} / \mathrm{Tag}$ ) ist mit geringerem Risiko für beginnenden Hypertonus verbunden (RR 0,54 gegenüber Teilnehmern mit Folsäureaufnahme von weniger als $200 \mathrm{mcg} / \mathrm{Tag})$. Prospektive Kohortenstudien Nurses Health Study II (93803 Teilnehmer) und Nurses Health Study I (62 260 Teilnehmer). 